\title{
A Numerical and Analytical Study of Phase Synchronization in Coupled SC-CNN Based Non-Identical Chaotic Systems
}

\author{
H. Shameem Banu ${ }^{1 *}$ P.S. Sheik Uduman ${ }^{2}$ \\ 1* Department of Physics, B.S.Abdur Rahman Crescent Institute of Science and Technology, Vandalur, \\ Chennai, India. \\ ${ }^{2}$ Department of Mathematics \& AS, B.S.Abdur Rahman Crescent Institute of Science and Technology, \\ Vandalur, Chennai, India \\ *Corresponding Author E-mail: sheikuduman@crescent.education
}

Received: 29.8.2021; Revised: 25.11.2021; Accepted: 27.11.2021

CSociety for Himalayan Action Research and Development

\begin{abstract}
This paper seeks to address the phase synchronization phenomenon using the drive-response concept, in our proposed model, State Controlled Cellular Neural Network (SC-CNN) based on variant of MuraliLakshmanan-Chua (MLCV) circuit. Using this unidirectionally coupled chaotic non autonomous circuits, we described the transition of unsynchronous to synchronous state, by numerical simulation method as well as the results are confirmed by solving explicit analytical solution. In this aspect, the system undergoes the new effect of phase synchronization (PS) phenomenon have been observed before complete synchronization (CS) state. To characterize these phenomena by the phase portraits and the time series plots. Also particularly characterize for PS by the method of partial Poincare section map using phase difference versus time, numerically and analytically. The study of dynamics involved in SC-CNN circuit systems, mainly applicable in the field of neurosciences and in telecommunication fields.
\end{abstract}

Keywords: SC-CNN; Non-autonomous circuit; unidirectional coupling; Phase Synchronization.

\section{Introduction}

Synchronization of chaotic dynamical systems is a process wherein two chaotic systems are coupled each other may synchronize to a common chaotic trajectory. The chaotic system is unpredictable, due to sensitive initial conditions, it diverge exponentially until they become completely uncorrelated (Pecora et al, 1990). Chaos synchronization is an important role play in the complex dynamical behavior in research communities because it traces the advances in secure communication systems (Boccaletti et al, 2002). Also, there are different kinds of networks, such as unidirectional networks, bidirectional networks, random networks, small-world networks and so on (Venkkatesh et al, 2016). In the context of chaos synchronization state have been studied in various methods incidentally, such as phase synchronization (PS) (Rosenblum et al, 1996, Parlitz et al, 1996), lag synchronization (LS), generalized synchronization, intermittent lag synchronization (ILS), imperfect phase synchronization 
(IPS) (Antonio Pujol-Pere et al, 2003), complete or identical synchronization (CS) and almost synchronization (AS) (Murali et al, 1996), (Srinivasan et al, 2016).

In this context, we proposed a chaotic nonidentical coupled nonautonomous circuits based on the ideas of Cellular Neural/nonlinear Network (CNN), which is an array of simple, interconnected nonlinear first order dynamical circuits, called cells introduced by Chua and Yang (Chua et al, 1988),. The main generalizations of CNN consist of direct dependence on neighborhood cells, named as State Controlled Cellular Neural Network (SC-CNNs). Basically, CNN consists of the most complex architecture, where analog has been converted by digital logic sections with low power consumption. This SC-CNN cell constitutes a second-order nonlinear circuit that, isolated from its local connectivity of cells and a fundamental simplicity. The feature of our proposed model is for implemented in many practical problems related to neural networks and generating several chaotic circuits, particularly suitable for image processing and spatial pattern formation (Arena et al, 1995). According to this aspect, the proposed chaotic nonidentical coupled nonautonomous circuits of dynamical system, is a simple forced parallel LCR circuit or named as variant of Murali-LakshmananChua (MLCV) circuit with the nonlinear element, Chua's diode, introduced by Thamilmaran (Thamilmaran et al, 2002), in the process of SC-CNN, showing routes to chaos, including periodic and aperiodic in nature (Gunay et al, 2010, Swathy et al, 2014). Among all these phenomena, our proposed chaotic systems are unidirectionally coupled, i.e., drive-response configuration. This shows that, the dynamics of the response system is to follow the dynamics of the drive system, while by increasing the strength of the coupling parameter, the systems transits from asynchronization to PS and then CS.

In this report, we focus mainly on the study of phase synchronization (PS) phenomenon using the generalized SC-CNN equation based MLCV circuit under unidirectionally coupled, by numerical simulation result and by an explicit analytical solution (Raissi et al, 2019). For the case of chaotic nonautonomous oscillator, the phase synchronization (PS) phenomenon originally introduced by Pikovsky (Rosenblum et al, 1996). This phase analysis concept has been successfully applied in biological neuron activities, cardiorespiratory system, optical topography and so on (Qingying Mino et al, 2009).

This paper is divided into five sections. In section 1, we present an introduction of this paper and in section 2, describe about the chaotic system of SC-CNN based MLCV. In section 3, gives the numerical results of phase and complete synchronization phenomena. An explicit analytical solution of generalized equations of our proposed model were carried out in section 4, and the final section 5, deals with conclusions. 


\section{Chaotic system description of SC-CNN based MLCV circuit}

This paper describes the dynamics of unidirectionally coupled chaotic nonidentical systems. In this connection, we draw on MLCV circuit presents a new approach to consider as a CNN structure of interconnected unit cells (Swathy et al, 2014, Chua et al, 1988). The SC-CNN described by the state equations (Arena et al, 1995):

$\dot{x}_{j}=-x_{j}+\sum_{C(k) \in N(j)} A_{j i k} y_{j}+\sum_{C(k) \in N(j)} B_{j j k} u_{j}+\sum_{C(k) \in N(j)} C_{j i k} x_{j}+I_{j}(1)$

Here $_{j}=f\left(x_{j}\right)=0.5\left(\left|x_{j}+1\right|-\left|x_{j}-1\right|\right)$.

where $j$ is the cell index, $x_{j}$ is the state variable. Using this state equations, we execute into two CNN cell as a first order differential equation based on MLCV circuit (Thamilmaran et al, 2000). We undertook this study to describe the synchronization of chaos in different methodology.

\section{Numerical Simulation Results}

Chaos synchronization of a coupled oscillator is a universal phenomenon that deals in science and engineering. Using coupling term, $\varepsilon$ of two nonautonomous self-sustained oscillators are set to interact. In this proposed model, SC-CNN based MLCV circuit equations of the drive and response systems are non-identical, by keeping, mismatch frequencies, $\left(\omega_{1}\right.$ and $\left.\omega_{2}\right)$ and an initial conditions, $\left(x_{10}, x_{20}, x_{30}, x_{40}\right)$, so that they give rise to different chaotic attractors when the coupling parameter, $\varepsilon$ is zero. Hence the two systems are naturally unsynchronized. Using Kirchhoff's law, the MLCV circuit equation can be written in the generalized SC-CNN equations has for the drive and the response system as shown below:

Drive system,

$\dot{x}_{1}=-x_{1}+a_{1} y_{1}+s_{11} x_{1}+s_{12} x_{2}+f_{1} \sin \omega_{1} t$

$\dot{x}_{2}=-x_{2}+s_{21} x_{1}+s_{22} x_{2}$

Response system,

$$
\begin{gathered}
\dot{x}_{3}=-x_{3}+a_{1} y_{3}+s_{11} x_{3}+s_{12} x_{4}+f_{2} \sin \omega_{2} t+\varepsilon\left(x_{1}-x_{3}\right) \\
\dot{x}_{4}=-x_{4}+s_{21} x_{3}+s_{22} x_{4}
\end{gathered}
$$

Where and are the output related to the state by the nonlinear equation,

$y_{1,3}=f\left(x_{1}, x_{3}\right)=0.5\left(\left|x_{1,3}+1\right|-\left|x_{1,3}-1\right|\right.$

Also, we fixed the system parameters such as, $a_{1}=0.6053, s_{11}=0.6047, s_{12}=-1.000, s_{21}=0.050$, $s_{22}=1.000$ and $\omega_{1,2}=0.105, f_{1}=0.411$ and $f_{2}=0.415 f_{1}=0.411, f_{2}=0.415$ and the initial conditions are fixed for the two systems as, $x_{10}=-0.5 ; x_{20}=0.1 ; x_{30}=0.5$ and $x_{40}=-0.11$. (Swathy et al, 2014). 
Therefore, Fig. (1) shows the chaotic attractors for the drive and the response systems in $\left(x_{1}-x_{3}\right)$ and $\left(x_{2}-x_{4}\right)$ phase planes respectively, for $\varepsilon=0.0$. As the coupling parameter, $\varepsilon$ is increased for a fixed mismatch frequency, $\left(\omega_{1}\right.$ and $\left.\omega_{2}\right)$ and an initial condition $\left(x_{10}, x_{20}, x_{30}, x_{40}\right)$, we observe the dynamics of an output undergoes unsynchronized state to synchronized state in the range between 0.0 $\geq \varepsilon \leq 2.0$. When the coupling parameter, increases gradually, then the dynamics of the drive is unaffected because of unidirectional coupling, only the response system varies according to the coupling parameter, $\varepsilon$. So that the chaotic attractor changes from the unsynchronized state to synchronized state of the drive and the response systems are shown in Fig. (2). In Fig. 2(a) and Fig. 2(b) shows the chaotic unsynchronized state of the drive and response systems in the $\left(x_{1}-x_{3}\right)$ phase space and their corresponding trajectory plot for $\varepsilon=0$. In Fig. 2(c) and Fig. 2(d), the chaotic synchronized state of the drive and response systems in the $\left(x_{1}-x_{3}\right)$ phase plane and the corresponding trajectory plot for $\varepsilon=1.0$.

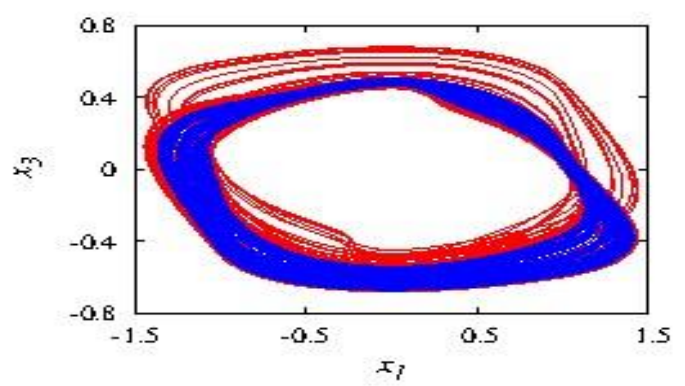

Figure 1: The frequency of the drive system is fixed as 0.411 (Red attractor), whereas the frequency of the response system is fixed as 0.415 (Blue attractor). Both the systems are fixed as non-identical chaotic attractors.

However, the proposed chaotic systems are non-identical, the drive and the response systems undergo phase synchronization (PS) in their dynamics, as the coupling parameter increases, which tends to a perfect matching of their phases, while the phase difference, $\Delta \varphi$ occurs in a random-walk type motion. So that, the phases of the response system $\varphi_{2}$, initially out of phase with the phases of the drive system $\varphi_{1}$ for small values of $\varepsilon$, but synchronizes in phase with the drive for the value of coupling parameter, $\varepsilon$. In these aspects, we study the PS phenomenon, using Runge-Kutta $4^{\text {th }}$ order for numerical simulation algorithm having step size, $h=0.1$ and by varying the control parameter exhibited coupled SC-CNN based MLCV circuit by unidirectionally. To characterize PS phenomenon by phase portrait in the $\left(x_{1}-x_{3}\right)$ phase planes and the trajectories plot for $\left(t, x_{1,3}\right)$ as shown in Fig. 3(a) and Fig. 3(b). 

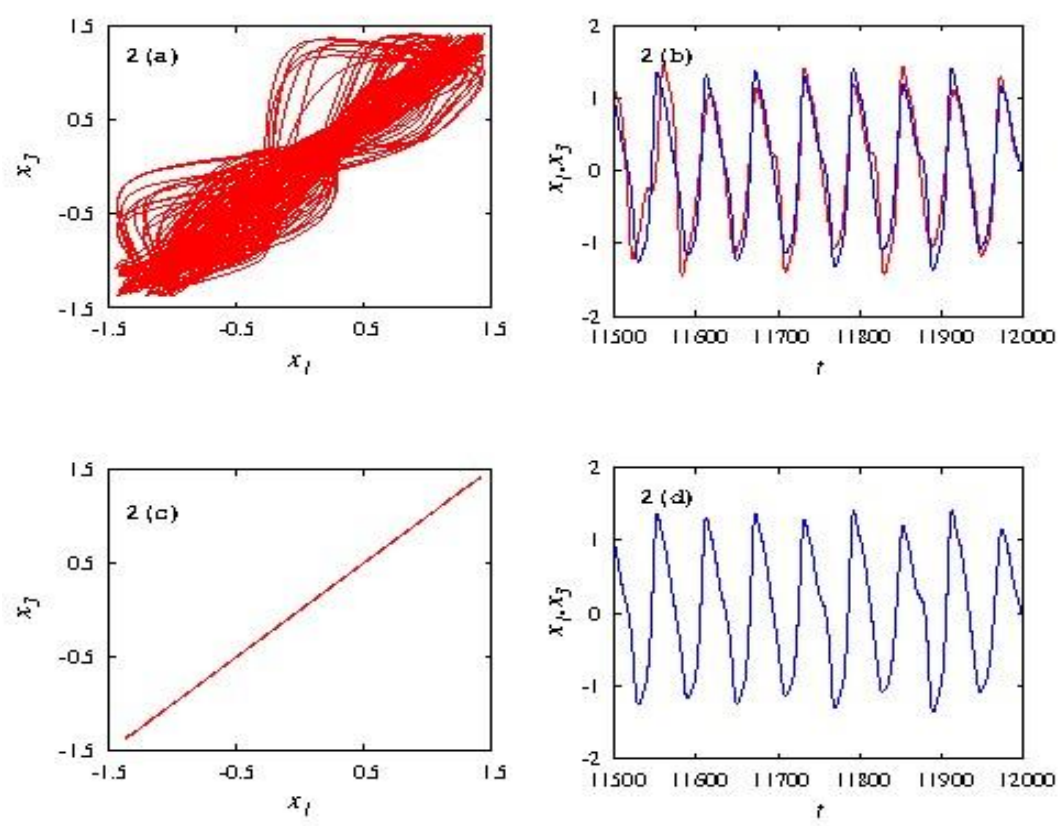

Figure 2: The phase space and their time series plot of an unsynchronized state of the two systems, shown in figs. 2(a) and 2(b), $\varepsilon=0.0$. For the synchronized state, shown in fig. 2(c) and 2(d), for $\varepsilon=1.0$.
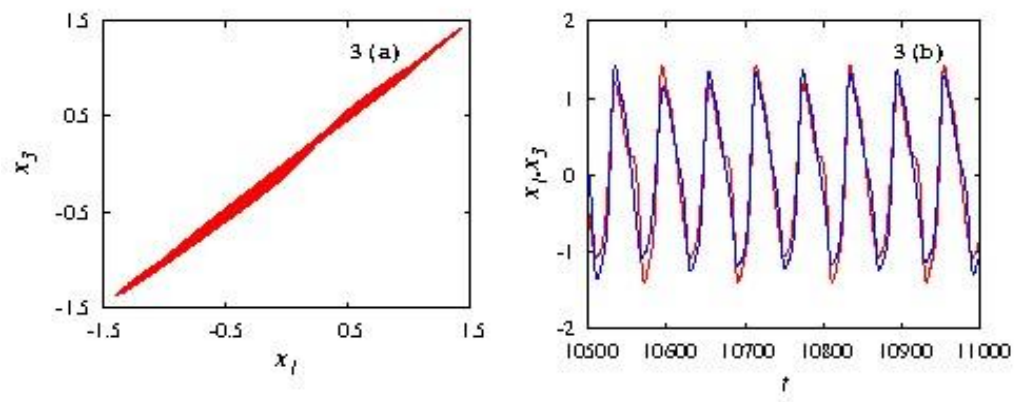

Figure 3: Phase synchronization occurs at $\varepsilon=0.035$, corresponding phase portrait(fig(3a)) and time series plot (fig $(3 b))$.

Further, for the confirmation of PS, we characterize by using Poincare section in a proper way, such that for each rotation, the $2 \pi$ phase increases, using the formula,

$$
\mathrm{M}=2 \pi \frac{\mathrm{t}-\mathrm{t}_{\mathrm{n}}}{\mathrm{t}_{\mathrm{n}+1}-\mathrm{t}_{\mathrm{n}}}+2 \pi \mathrm{n}, \quad \mathrm{t}_{\mathrm{n}} \leq \mathrm{t}<\mathrm{t}_{\mathrm{n}+1}(4)
$$

Where $t_{n}$ is the time of the $n^{\text {th }}$ crossing of the secant surface. For the numerical simulation results of the two systems are completely locked for the coupling strength $\varepsilon=0.0048$, as shown in Fig. (4). 


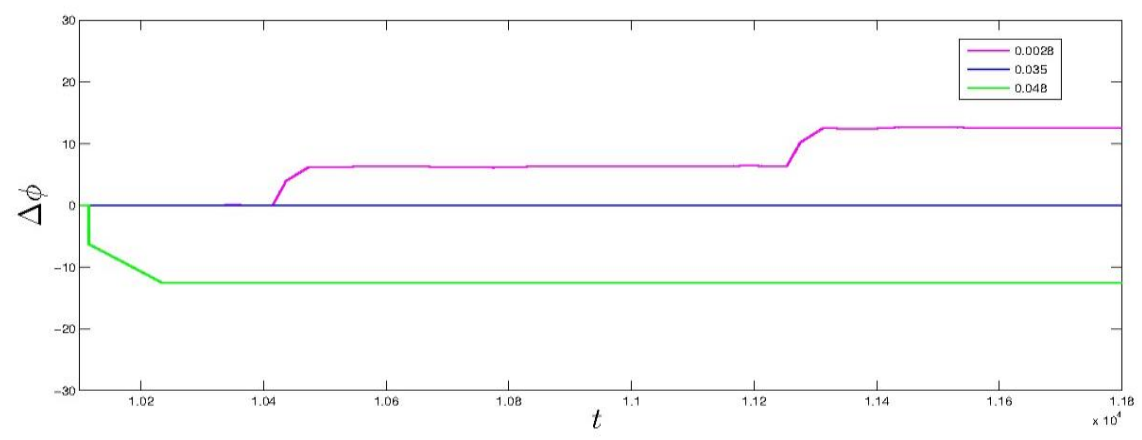

Figure 4: Phase synchronization occurs at $\varepsilon=0.035$ (blue line), from an analytical result, inwhich phase difference is in constant. Imperfect phase synchronization also shown for $\varepsilon=0.0028$ (magenta line) and $\varepsilon=0.048$ (green line).

From the figure, we have obtained PS, while the phase differences do not grow with time ie., $\Delta \varphi=0$.

This numerical simulation result has further strengthened by analyzing analytically, as follows:

\section{Explicit Analytical Results}

To exemplify the dynamical behavior analytically, by combining the drive and the response system equations (2) and (3), form a difference equation, by considering $\left.x_{1}^{*}=\left(x_{1}-x_{3}\right) ; x_{2}^{*}=\left(x_{2}-x_{4}\right)\right)$; $y_{1}^{*}=\left(y_{1}-y_{3}\right)$ as follows:

$\dot{x}_{1}^{*}=-x_{1}^{*}+a_{1} y_{1}^{*}+s_{11} x_{1}^{*}+s_{12} x_{2}^{*}+f_{1} \sin \omega_{1} t-f_{2} \sin \omega_{2} t-\varepsilon x_{1}^{*}$

$\dot{x}_{2}^{*}=-x_{2}^{*}+s_{21} x_{1}^{*}+s_{22} x_{2}^{*}$

Where $y_{1}^{*}=f\left(x_{1}^{*}\right)=\left\{\begin{array}{cc}1, & x_{1}^{*} \geq 1, \\ x_{1}^{*}, & x_{1}^{*} \leq 1, \\ 1, & x_{1}^{*} \leq-1 .\end{array}\right.$

Here, $y_{1}^{*}$ represents the nonlinear term, considered as the three piecewise linear regions $D_{0}, D_{ \pm 1}$ as per CNN model. By fixing coupling parameter, $\varepsilon=0$, then the state variables $x_{1}(t)$ and $x_{2}(t)$ has an explicit analytical solution and considered as the unsynchronized state.

For an analytical solution, the frequencies of the two systems are fixed as chaotic attractors $(0.411$ for drive system and 0.415 for response system), as shown in Fig. (5). 


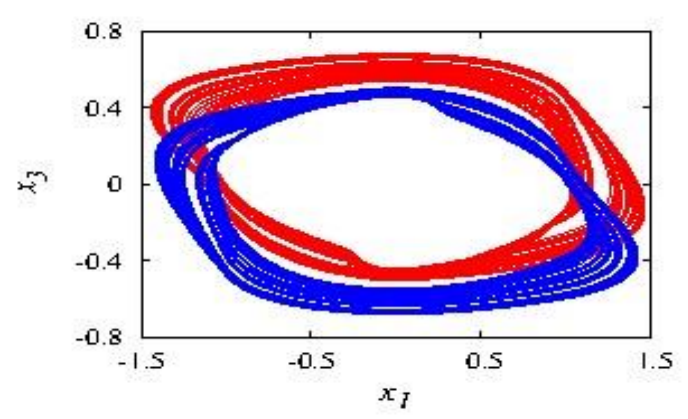

Figure 5: The frequency of the drive system is fixed as 0.411 (Red attractor), whereas the frequency of the response system is fixed as 0.415 (Blue attractor). Both the systems are fixed as non-identical chaotic attractors.

Now, by changing the coupling parameter, i.e., $(\varepsilon>0)$, then the coupled system reaches the synchronized state. Hence, the solution for the difference equations in two different cases for each of three piecewise linear regions $D_{0}, D_{ \pm 1}$ as follows: For $D_{0}$ region, when the coupling parameter, $\varepsilon=0.56821$, then the roots become complex conjugate, an explicit analytical solution for the state variables $x_{2}(\mathrm{t})$ and $x_{1}(\mathrm{t})$ are,

$$
\begin{aligned}
x_{2}^{*}(t)=e^{\alpha_{1} t}\left[C_{1} \cos \alpha_{2} t+C_{2} \sin \alpha_{2} t\right]+E_{1} \sin \omega_{1} t+E_{2} \cos \omega_{1} t \\
+E_{3} \sin \omega_{2} t+E_{4} \cos \omega_{2} t \\
x_{1}(t)=\frac{1}{s_{21}}\left\{e ^ { \alpha _ { 1 } t } \left[C_{1}\left(\left(\alpha_{1}+1-s_{22}\right) \cos \alpha_{2} t-\alpha_{2} \sin \alpha_{2} t\right)\right.\right. \\
\left.+C_{2}\left(\alpha_{2} \cos \alpha_{2} t+\left(\alpha_{1}+1-s_{22}\right) \sin \alpha_{2} t\right)\right]+E_{1}\left[\omega_{1} \cos \omega_{1} t\right. \\
\left.+\sin \omega_{1} t\left(1-s_{22}\right)\right]+E_{2}\left[\cos \omega_{1} t\left(1-s_{22}\right)-\omega_{1} \sin \omega_{1} t\right] \\
+E_{3}\left[\omega_{2} \cos \omega_{2} t+\sin \omega_{2} t\left(1-s_{22}\right)+E_{4}\left[\cos \omega_{2} t\left(1-s_{22}\right)\right.\right. \\
\left.\left.-\omega_{2} \sin \omega_{2} t\right]\right\}
\end{aligned}
$$

Here $E_{1}, E_{2}, E_{3}, E_{4}$ are called as arbitrary constants and $C_{1,2}$ are combination/integration constants, which can be calculated by keeping the initial conditions $x_{10}^{*}$ and $x_{20}^{*}$ at time $t_{0}$. Thus, $C_{1}$ and $C_{2}$ can be obtained as, 


$$
\begin{aligned}
C_{1}=\frac{e^{-\alpha_{1} t_{0}}}{\alpha_{2}}[ & \alpha_{2} x_{20}^{*} \cos \alpha_{2} t_{0}-x_{10}^{*}\left(s_{21}-\alpha_{1}\right) \sin \alpha_{2} t_{0} \\
& +\left(\left(E_{1} \omega_{1}-E_{2} \alpha_{1}\right) \sin \alpha_{2} t_{0}-\left(E_{2} \alpha_{2}\right) \cos \alpha_{2} t_{0}\right) \cos \omega_{1} t_{0} \\
& -\left(\left(E_{1} \alpha_{1}+E_{2} \omega_{1}\right) \sin \alpha_{2} t_{0}+\left(E_{1} \alpha_{2}\right) \cos \alpha_{2} t_{0}\right) \sin \omega_{1} t_{0} \\
& +\left(\left(E_{3} \omega_{2}-E_{4} \alpha_{1}\right) \sin \alpha_{2} t_{0}-\left(E_{4} \alpha_{2}\right) \cos \alpha_{2} t_{0}\right) \cos \omega_{2} t_{0} \\
& \left.-\left(\left(E_{3} \alpha_{1}+E_{4} \omega_{2}\right) \sin \alpha_{2} t_{0}+\left(E_{3} \alpha_{2}\right) \cos \alpha_{2} t_{0}\right) \sin \omega_{2} t_{0}\right] \\
C_{2}=\frac{e^{-\alpha_{1} t_{0}}}{\alpha_{2}}[ & \alpha_{2} x_{20}^{*} \sin \alpha_{2} t_{0}-x_{10}^{*}\left(s_{21}-\alpha_{1}\right) \cos \alpha_{2} t_{0} \\
& -\left(\left(E_{1} \omega_{1}-E_{2} \alpha_{1}\right) \cos \alpha_{2} t_{0}+\left(E_{2} \alpha_{2}\right) \sin \alpha_{2} t_{0}\right) \cos \omega_{1} t_{0} \\
& +\left(\left(E_{1} \alpha_{1}+E_{2} \omega_{1}\right) \cos \alpha_{2} t_{0}-\left(E_{1} \alpha_{2}\right) \sin \alpha_{2} t_{0}\right) \sin \omega_{1} t_{0} \\
& -\left(\left(E_{3} \omega_{2}-E_{4} \alpha_{1}\right) \cos \alpha_{2} t_{0}+\left(E_{4} \alpha_{2}\right) \sin \alpha_{2} t_{0}\right) \cos \omega_{2} t_{0} \\
& \left.+\left(\left(E_{3} \alpha_{1}+E_{4} \omega_{2}\right) \cos \alpha_{2} t_{0}-\left(E_{3} \alpha_{2}\right) \sin \alpha_{2} t_{0}\right) \sin \omega_{2} t_{0}\right]
\end{aligned}
$$

Using eqns. (6) and (7), we can calculate $x_{3}(t)$ and $x_{4}(t)$ using the relations $x_{3}(t)=x_{1}(t)-x_{1}^{*}(t)$ ; $x_{4}(t)=x_{2}(t)-x_{2}^{*}(t)$ and for $\varepsilon \geq 0.56821$, then the rootsare real and distinct. Therefore, the general solution becomes,

$$
\begin{array}{r}
x_{2}^{*}(t)=C_{1} e^{\alpha_{1} t}+C_{2} e^{\alpha_{2} t}+E_{1} \sin \omega_{1} t+E_{2} \cos \omega_{1} t \\
+E_{3} \sin \omega_{2} t+E_{4} \cos \omega_{2} t(8) \\
x_{1}(t)=\frac{1}{s_{21}}\left\{e ^ { \alpha _ { 1 } t } \left[C _ { 1 } \left(\left(\alpha_{1}+1-s_{22}\right) \cos \alpha_{2} t-\alpha_{2} \sin \alpha_{2} t\right.\right.\right. \\
\left.+C_{2}\left(\alpha_{2} \cos \alpha_{2} t+\left(\alpha_{1}+1-s_{22}\right) \sin \alpha_{2} t\right)\right] \\
+E_{1}\left[\omega_{1} \cos \omega_{1} t+\sin \omega_{1} t\left(1-s_{22}\right)\right] \\
+E_{2}\left[\cos \omega_{1} t\left(1-s_{22}\right)-\omega_{1} \sin \omega_{1} t\right] \\
+E_{3}\left[\omega_{2} \cos \omega_{2} t+\sin \omega_{2} t\left(1-s_{22}\right)\right] \\
\left.+E_{4}\left[\cos \omega_{2} t\left(1-s_{22}\right)-\omega_{2} \sin \omega_{2} t\right]\right\}
\end{array}
$$

The constants $C_{1}$ and $C_{2}$ can be evaluated by solving the eqn. (5) at time $t_{0}$.

$$
\begin{aligned}
& C_{1}=\frac{e^{-\alpha_{1} t_{0}}}{\alpha_{2}-\alpha_{1}}\left[\left(s_{21} x_{10}^{*}-\alpha_{1} x_{20}^{*}\right)+\left(\left(E_{2} \omega_{1}+E_{1} \alpha_{1}\right) \sin \omega_{1} t_{0}+\right.\right. \\
& \left(E_{2} \alpha_{1}-E_{1} \omega_{1}\right) \cos \omega_{1} t_{0}+\left(E_{3} \alpha_{2}+E_{4} \omega_{2}\right) \sin \omega_{2} t_{0}+ \\
& \left(E_{4} \alpha_{2}-E_{3} \omega_{2}\right) \cos \omega_{2} t_{0}
\end{aligned}
$$




$$
\begin{aligned}
& C_{2}=\frac{e^{-\alpha_{1} t_{0}}}{\alpha_{1}-\alpha_{2}}\left[\left(s_{21} x_{10}^{*}-\alpha_{2} x_{20}^{*}\right)+\left(\left(E_{2} \omega_{1}+E_{1} \alpha_{2}\right) \sin \omega_{1} t_{0}+\right.\right. \\
& \left(E_{2} \alpha_{2}-E_{1} \omega_{1}\right) \cos \omega_{1} t_{0}+\left(E_{3} \alpha_{1}+E_{4} \omega_{2}\right) \sin \omega_{2} t_{0}+\left(E_{4} \alpha_{1}-\right. \\
& \left.E_{3} \omega_{2}\right) \cos \omega_{2} t_{0}
\end{aligned}
$$

Using the above equations, we can calculate $x_{3}(\mathrm{t})$ and $x_{4}(\mathrm{t})$. For $D_{ \pm 1}^{*}$ regions, the nonlinearity function is taken as 1 . Hence the generalized equations are obtained as,

$$
\begin{aligned}
& \dot{x}_{1}^{*}=\left(s_{11}-\varepsilon-1\right) x_{1}^{*}+s_{12} x_{2}^{*}+f_{1} \sin \omega_{1} t-f_{2} \sin \omega_{2} t \\
& \dot{x}_{2}^{*}=-x_{2}^{*}+s_{21} x_{1}^{*}+s_{22} x_{2}^{*}
\end{aligned}
$$

For $\varepsilon<0.05191$,

$$
\begin{gathered}
x_{2}^{*}(t)=e^{\alpha_{\mathrm{g}} t}\left[C_{3} \cos \alpha_{4} t+C_{4} \cos \alpha_{4} t\right]+E_{5} \sin \omega_{1} t+E_{6} \cos \omega_{1} t \\
+E_{7} \sin \omega_{2} t+E_{8} \cos \omega_{2} t
\end{gathered}
$$

The constants $E_{5}, E_{6}, E_{7}, E_{8}$ in $D_{0}^{*}$ region, are the same as the constants $E_{1}, E_{2}, E_{3}, E_{4}$, except that the constants A \& B are renew with C \& D respectively.

$$
\begin{aligned}
& x_{1}^{*}(t)=\frac{1}{s_{21}}\left\{e ^ { \alpha _ { \mathrm { g } } t } \left[C_{3}\left(\left(\alpha_{3}+1-s_{22}\right) \cos \alpha_{4} t-\alpha_{4} \sin \alpha_{4} t\right)\right.\right. \\
& \left.\quad+C_{4}\left(\alpha_{4} \cos \alpha_{4} t+\left(\alpha_{3}+1-s_{22}\right) \sin \alpha_{4} t\right)\right]
\end{aligned}
$$

The constants $C_{3}$ and $C_{4}$ are the same as $C_{1}$ and $C_{2}$ in case (i) of $D_{0}^{*}$ region except that the constants $E_{1}$, $E_{2}, E_{3}, E_{4}$ are replaced with the constants $E_{5}, E_{6}, E_{7}, E_{8}$ respectively. From the results of $x_{2}^{*}(t), x_{1}^{*}(t)$,we can evaluate $x_{1}(\mathrm{t}), x_{2}(\mathrm{t}), x_{3}(\mathrm{t})$ and $x_{4}(\mathrm{t})$.

For $\varepsilon \geq 0.05191$, the roots are real and distinct. Then the general solution can be written as,

$$
\begin{gathered}
x_{2}^{*}(t)=C_{3} e^{\alpha_{\mathrm{g}} t}+C_{4} e^{\alpha_{4} t}+E_{5} \sin \omega_{1} t+E_{6} \cos \omega_{1} t \\
+E_{7} \sin \omega_{2} t+E_{8} \cos \omega_{2} t
\end{gathered}
$$

where $C_{3}$ and $C_{4}$ are the integration constants and the constants $E_{5}, E_{6}, E_{7}, E_{8}$ are the same as for $D_{0}$ region.

$$
\begin{array}{r}
x_{1}^{*}(t)=\frac{1}{s_{21}}\left\{e ^ { \alpha _ { 3 } t } \left[C_{1}\left(\left(\alpha_{3}+1-s_{22}\right) \cos \alpha_{4} t-\alpha_{4} \sin \alpha_{4} t\right)\right.\right. \\
\left.+C_{2}\left(\alpha_{4} \cos \alpha_{4} t+\left(\alpha_{3}+1-s_{22}\right) \sin \alpha_{4} t\right)\right]
\end{array}
$$




$$
\begin{aligned}
& +E_{5}\left[\omega_{1} \cos \omega_{1} t+\sin \omega_{1} t\left(1-s_{22}\right)\right] \\
& +E_{6}\left[\cos \omega_{1} t\left(1-s_{22}\right)-\omega_{1} \sin \omega_{1} t\right] \\
& +E_{7}\left[\omega_{2} \cos \omega_{2} t+\sin \omega_{2} t\left(1-s_{22}\right)\right] \\
& \left.+E_{8}\left[\cos \omega_{2} t\left(1-s_{22}\right)-\omega_{2} \sin \omega_{2} t\right]\right\}
\end{aligned}
$$

The constants $C_{3}$ and $C_{4}$ are the same as $C_{1}$ and $C_{2}$ of $D_{0}^{*}$ except that the constants $E_{1}, E_{2}, E_{3}, E_{4}$ are changes as $E_{5}, E_{6}, E_{7}, E_{8}$ respectively. From the results, we can evaluate $x_{3}(\mathrm{t})$ and $x_{4}(\mathrm{t})($ Shameem Banu et al, 2018).

From the above aspects of the three regions $D_{0}, D_{+}$and $D_{-}$solutions, we can generate the characteristics, such as phase portraits, time series plot and Poincare section map. Using this remarkable result to emerge from the data for a particular value of coupling parameter, eto get an unsynchronized state, phase synchronization and a chaotic synchronized state. These characteristics revealed in Fig. (6). For an unsynchronized state (fig. 6(a) and fig. 6(b), $\varepsilon=0.0$, so that the two systems, drive and response are not coupled, acts as an independent system, whereas for the chaotic synchronized state (fig.6(c) and fig. 6(d)) for $\varepsilon=3.2$.
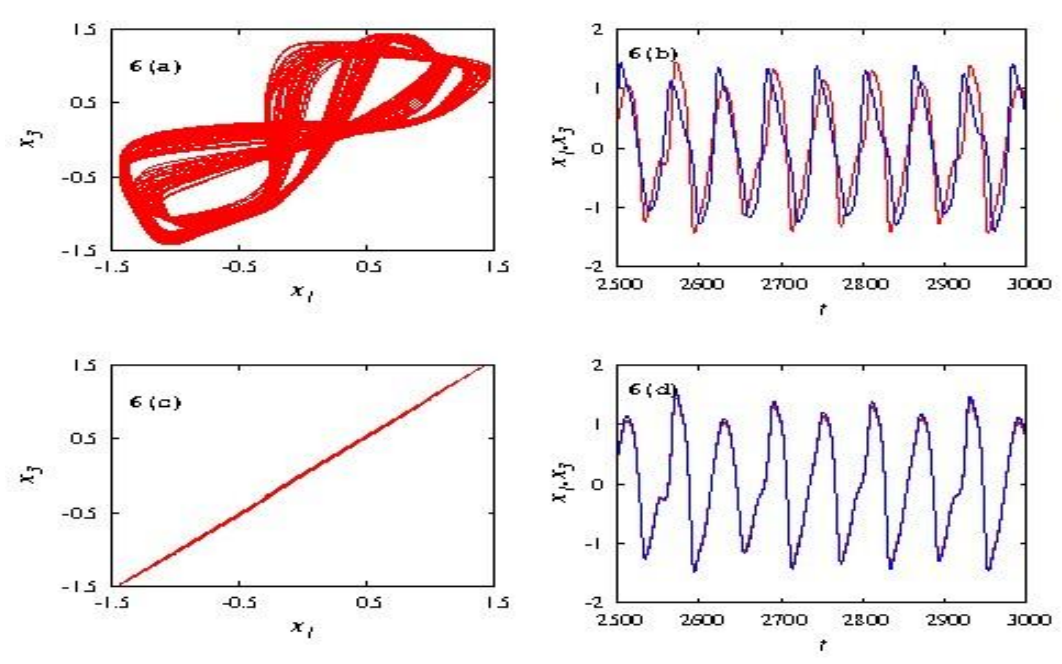

Figure 6: The phase space and their time series plot from an analytical result, for an unsynchronized state of the two systems, shown in figs. 6(a) and 6(b), $\varepsilon=0.0$. For the synchronized state, shown in fig. 6(c) and 6(d), for $\varepsilon=3.2$.

For the study of phase synchronization phenomena using analytical solution, for the value of $\varepsilon=1.5$, the phase portraits and their corresponding time trajectories are shown in Fig. 7(a) and Fig. 7(b). 

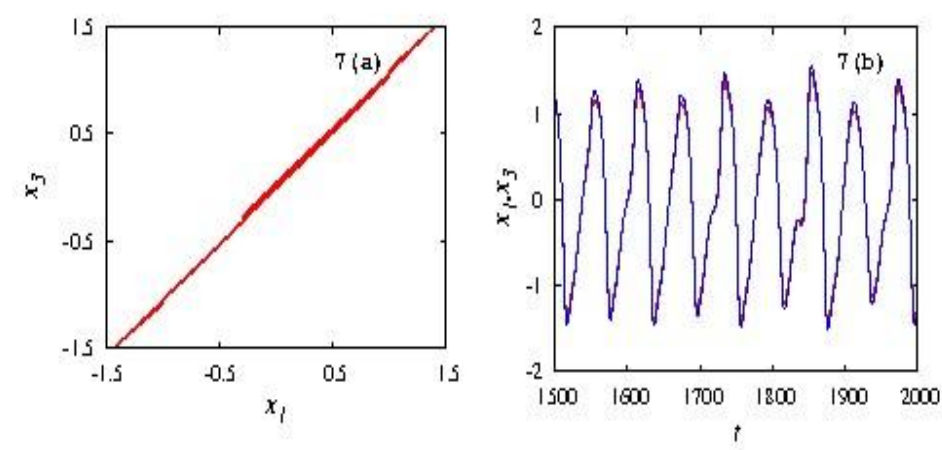

Figure 7: Phase synchronization occurs at $\varepsilon=1.5$, corresponding phase portrait (fig(7a)) and time series plot (fig(7b)).

Also, from the same analytical data applied in the Poincare section formula (Eqn. (4)), is to characterize the phase synchronization phenomena analytically, as shown in Fig. (8).

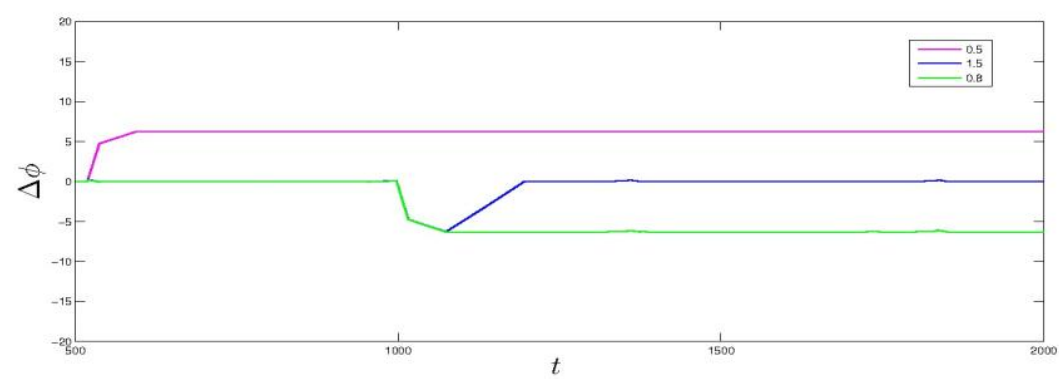

Figure 8: Phase synchronization occurs at $\varepsilon=1.5$ (blue line), from an analytical result, inwhich phase difference is in constant. Imperfect phase synchronization also shown for $\varepsilon=0.5$ (magenta line) and $\varepsilon=0.8$ (green line).

From the above figure, the PS phenomenon is confirmed by plotting these characteristics, using the analytically solved data. The phase difference, $\Delta \varphi$ must be in constant, ie., does not grow with time, in analytical results, Fig. (8), which is coincide with the numerical results, as in Fig. (4).

\section{Conclusion}

In this paper, we have presented numerically and an explicit analytical study on unidirectional coupled, non-identical two generalized SC-CNN cell equations, based MLCV circuit systems exhibiting for the chaotic synchronization phenomena. Especially, our research has highlighted the importance of phase synchronization in a chaotic coupled system, in which we have confirmed further evidence for the PS using Poincaresectional map for numerical result as well as for an analytical result. According to the detection of phase synchronization, in our proposed model, SC-CNN of a unidirectional chaotic coupled system, is suitable for the neuron activities and in signal 
communications. Also, this could be eventually led to the application of nephron-nephron interaction in future.

\section{Acknowledgement}

We would like to thank, Dr. K. Thamilmaran, and Dr. Leo Kingston for their support, without whose help this work would never have been possible.

\section{References}

Antonio Pujol-Pere, Oscar Calvo, Manuel A. Matias, Jurgen Kurths (2003) Experimental study of imperfect phase synchronization in the forced Lorenz system, Chaos, 13, 319-326.

Arena P, Baglio S, Fortuna L, Manganaro G (1995) Chua's circuit can be generated by CNN cells. IEEE Trans. Circuits Syst. I, 42, 123-125.

Boccaletti S, Kurths J, Osipov G, Valladares DL, Zhou CJ (2002) The Synchronization of chaotic systems. Physics Reports, 366, 1-101.

ChuaLO, Yang L (1988) Cellular neural networks:Theory”, IEEE Trans. Circuits Syst., 35, 12571272.

Gunay e (2009) MLC circuit in the frame of CNN.Int. J. Bifurcation and Chaos, 20, 3267-3274.

Murali K, Lakshmanan M (1996) Chaos in Nonlinear Oscillators: Controlling and Synchronization. World Scientific Series on Nonlinear Science, Singapore.

Parlitz U, Junge L, and Lauterborn W (1996) Experimental observation of phase synchronization.Phys. Rev. E, 54, 2115-2117.

Pecora LM, Carroll TL (1990) Synchronization in chaotic systems. Phys. Rev. Lett., 64, 821.

Qingying Miao, Yang Tang, Suojun Lu, and Jianan Fang (2009) Lag synchronization of a class of chaotic systems with unknown parameters. Nonlinear Dynamics, 57, 107-112.

Rosenblum MG,Pikovsky AS, and Jurgen Kurths (1996) Phase synchronization of chaotic oscillators.Phys. Rev. Lett., 76, pp. 1804-1807.

Raissi M, Perdikaris P, Karniadakis GE (2019) Physics-informed neural networks: A deep learning framework for solving forward and inverse problems involving nonlinear partial differential equations. Journal of Computational Physics, 378, 686-707.

Saeed Taherion, and Ying-Cheng Lai (1999) Observability of lag synchronization of coupled chaotic oscillators. Phys. Rev. E, 59.

Shameem Banu H, andSheik Uduman PS (2018) Chaos synchronization in coupled SCCNN based variant of MLC circuit: An analytical study. IEEE conference. 
Srinivasan K, Chandrasekar VK, Gladwin Pradeep R, Murali K, Lakshmanan M (2016) Different types of synchronization in coupled network based chaotic circuits. Communications in Nonlinear science and Numerical simulation.

Swathy PS, Thamilmaran K (2014) Dynamics of SC-CNN based variant of MLC circuit: An experimental study, Int. J. Bifurcation and Chaos, 24(2).

Thamilmaran K,Lakshmanan M, Murali K (2000) Rich variety of bifurcations and chaos in a variant of Murali-Lakshmanan-Chua circuit. Int. J. Bifurcation and Chaos, 10(7), 1175-1180.

Thamilmaran K, Lakshmanan M (2002) Classification of Bifurcations and Routes to Chaos in a Variant of Murali-Lakshmanan-Chua circuit. Int. J. Bifurcation and Chaos, 12(4), 783-813.

Venkatesh PR, Venkatesan A, Lakshmanan M (2016) Analytical treatment for synchronizing chaos through unidirectional coupling and implementation of logic gates. Pramana J. Phys., 86(6), 1195-1207. 